\title{
Design of the GSM Module Interface Circuit for the Intelligent Managerial System of Blasting Equipment Library
}

\author{
Xingshan $\mathrm{LI}^{1, \text { a }}$, Luhua Zhao ${ }^{2}$ \\ ${ }^{1}$ Luohe medical college, Luohe, 462000, China; \\ ${ }^{2}$ Henan quality engineering vocational college, Pingdingshan,475000, China \\ aemail:604141388@qq.com
}

Keywords: Coal Mine Blasting Equipment Library; Intelligence; Single Chip Microcomputer (SCM); GSM Module Interface Circuit

\begin{abstract}
This paper mainly analyses the necessity to manage the explosive in the coal mine blasting equipment library. It make intelligent management possible by using digital monitoring technology, communication technology and software technology and sensor technology for reference; using high performance microcontroller to identify if the fingerprint is legitimate; then control the opening and closing of the electronic lock.
\end{abstract}

\section{Introduction}

In order to ensure the safety in the production of coal mine, the storage of powder and its access management must be strict and intelligent. With the rapid development of digital control technology, we can make intelligent management possible by using the electronic lock and the fingerprint machine.

\section{Component of Coal Mining Blasting Equipment Library System}

The intelligent management system is made up of three parts: the lower machine, the control device and PC. The system is shown as Fig.1.

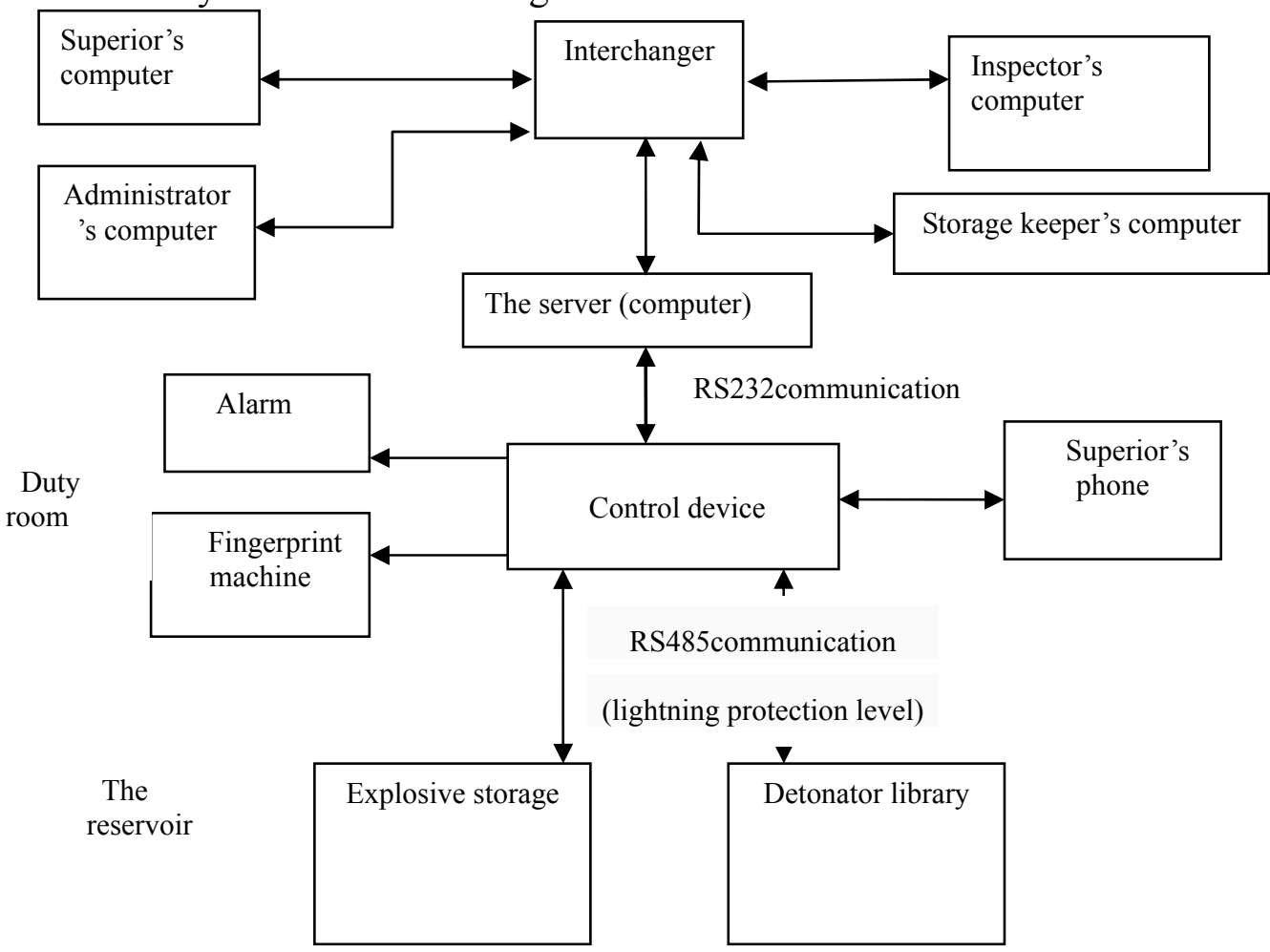

Fig.1. Managerial system of the coal mining blasting equipment library 


\section{Control System of the Lower Machine}

The storage and access of the explosives and detonators in the reservoir area are managed by the lower machine (computer entrance guard system). The control system of lower machine is mainly composed of single chip microcomputer STC12C5A60S, temperature and humidity sensor, vibration sensor, the fingerprint machine, electromagnetic lock control and communication module. The lower machine uses single chip to control the system [1]. The system is shown as Fig.2.

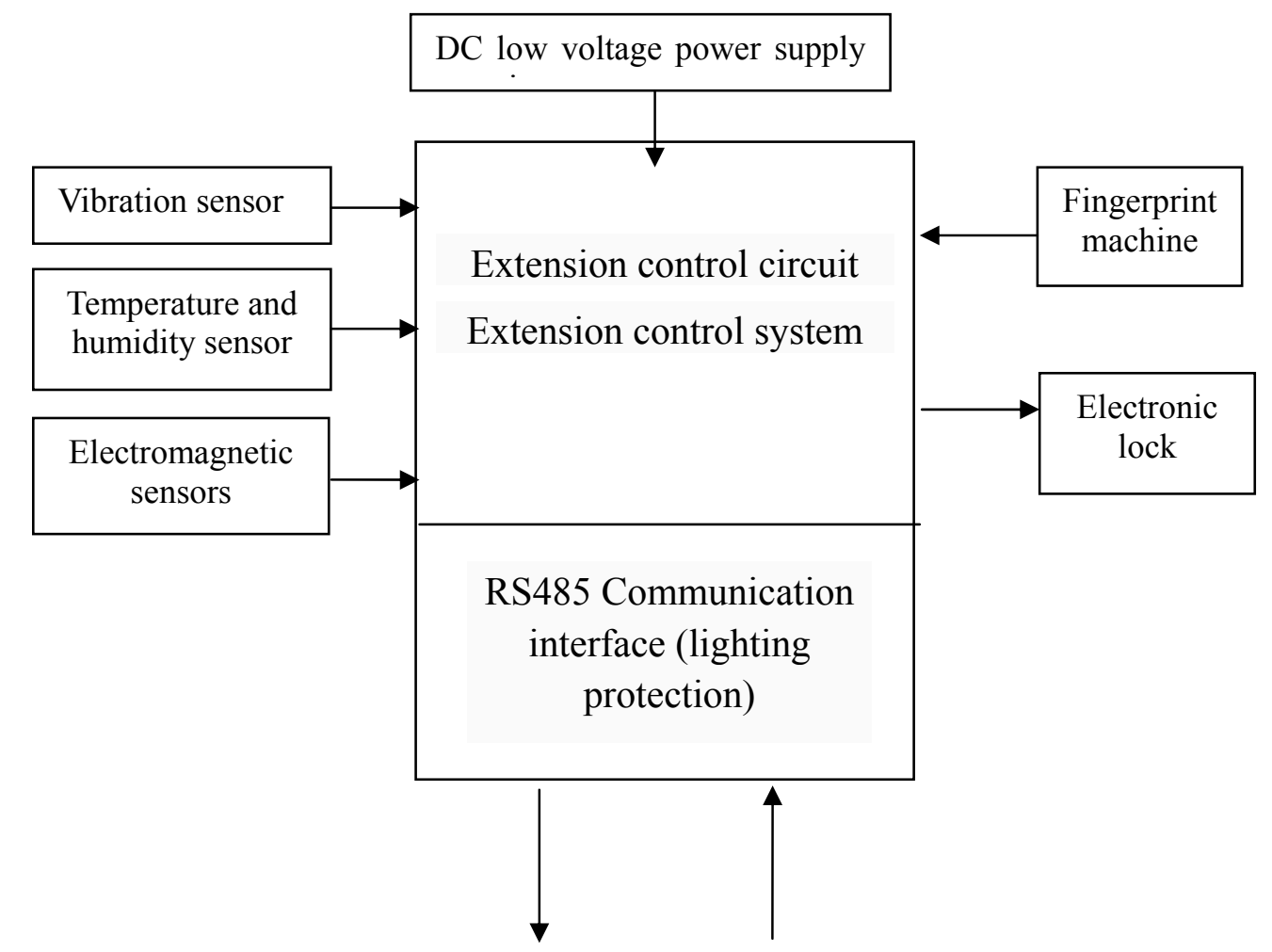

Fig.2. Control system of lower machine for coal mining blasting equipment library

\section{The Implementation of the GSM Module Interface Circuit}

When the lower machine of the blasting equipment library detects the excess of the temperature, humidity or air pressure, it will send an alarm message to the upper machine; at the same time, it will send a prompt alarm message to the cell phone number designated by the management system of the upper machine sending a text message alarm prompt. GSM module utilizes USES a phone card and cooperates with the cell phone card.

TC35 module is a GSM module which supports the reception and sending of Chinese GSM short messages and voice communication function. The working voltage between $3.3 \mathrm{~V}$ and $4.8 \mathrm{~V}$. The Module must be connected to SIM card and the antenna while working [2]. So the program development of TC35 module must firstly build the circuit system of the TC35 module, mainly including power supply, antenna and SIM card circuit. The communication between Single chip microcomputer and TC35 module is also accomplished through the serial port communication. The sending, receiving and reading of data of the TC35 module is achieved according to the AT command set. The modules can send and receive short messages in accordance with TEXT or PDU format. As a result, it is necessary to determine before sending messages[3]. In addition to the antenna interface, TC3 modul has 40 pins, which are through the zero-resistance socket connector. As the pins are relatively dense, attention must be paid to the circuit board processing technology. The 40 pins include there is power, the serial data port, SIM card interface, audio interface and control interface [4]. In the use of TC35 module, attention should be paid to the stability of the power supply; it is recommended to use $4.2 \mathrm{~V} \mathrm{dc}$ power supply as the signal strength is higher and the debugging is convenient. The working of the TC35 module undergoes a process of 
net work and it must be ensured that the TC35 module will send a low pulse signal after electrification to the IGT which is greater than 100 milliseconds and less than 1000 milliseconds. The 18th and 19th pins adopt TTL pins in serial communication and can be directly connected to the microcontroller serial port directly [5].

Single chip microcomputer system and TC35 module interface circuit is shown in figure 3 and figure 4 .

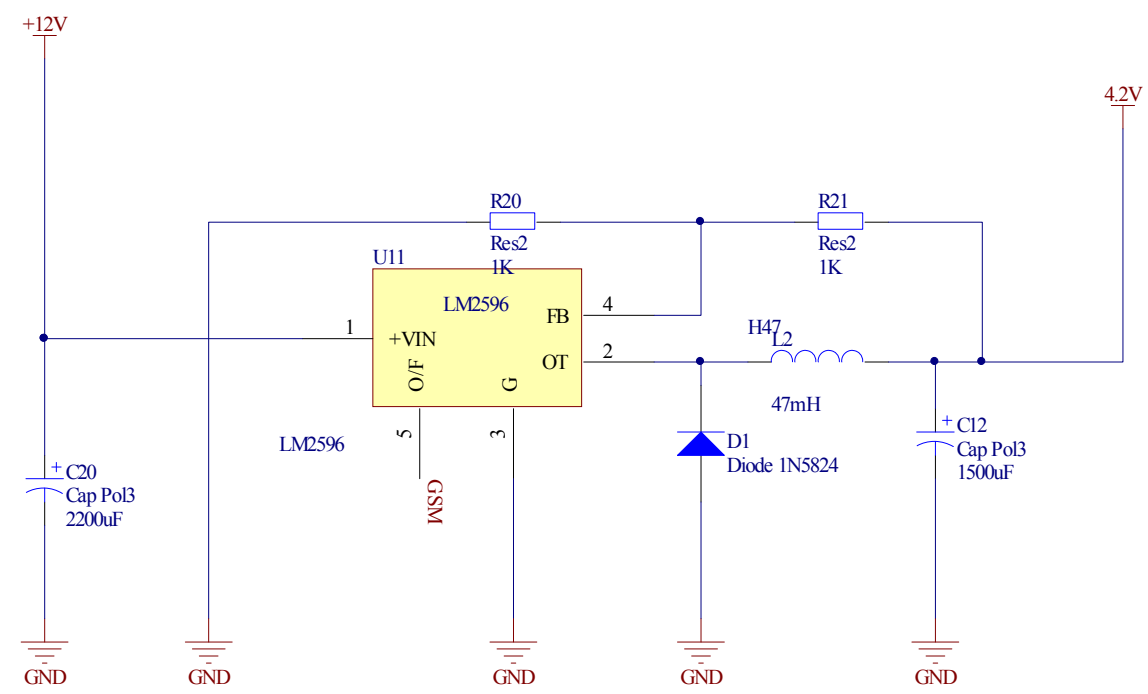

Fig. 3. Single chip microcomputer and TC35 module interface circuit(1) 


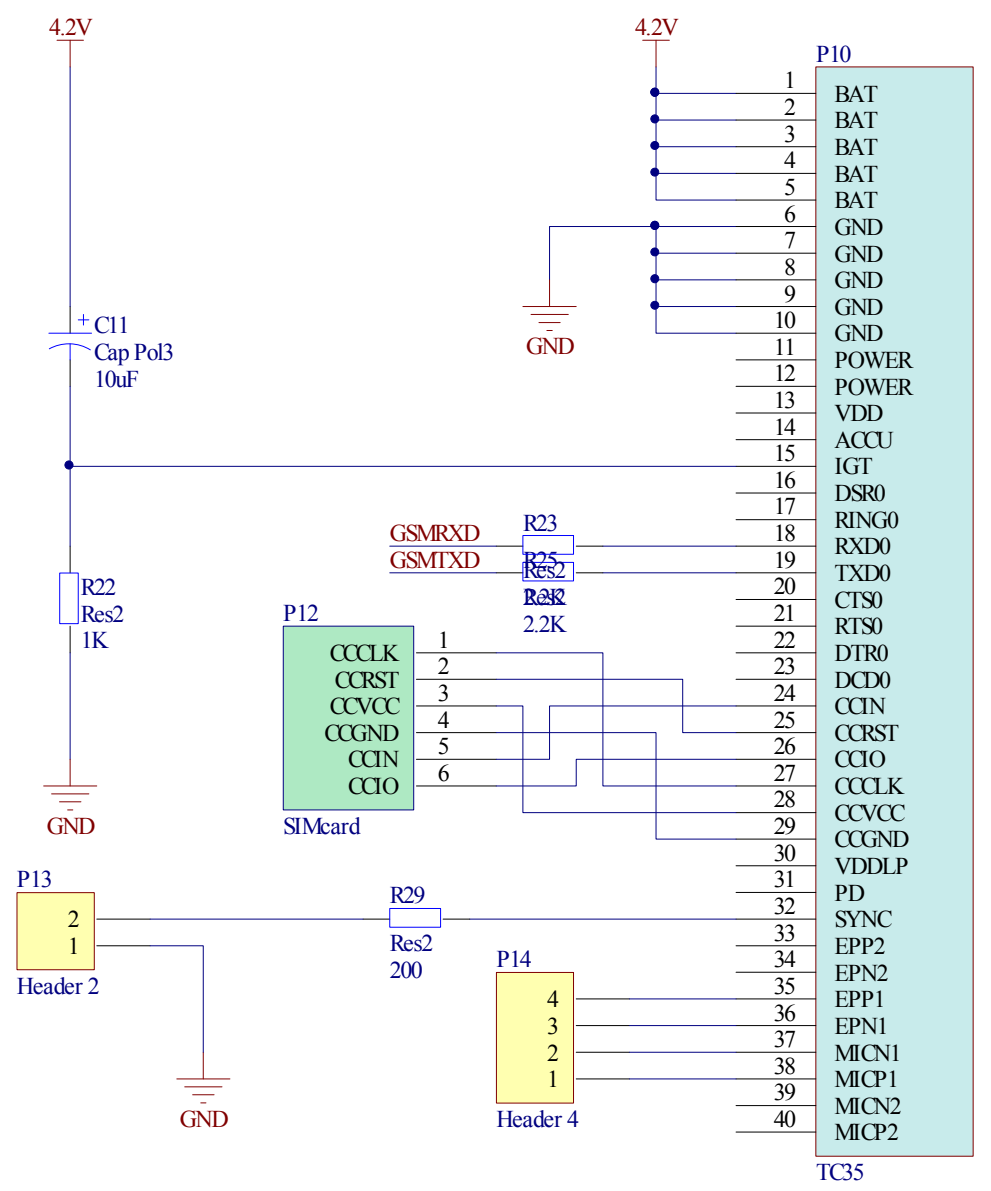

Fig. 4. Single chip microcomputer and TC35 module interface circuit(2)

\section{Conclusion}

Through reasonable design and a lot of experiments of the GSM module interface circuit, the experiment parameters of coal mining blasting equipment library can reach the expected indicators, avoid safety accidents caused by explosives from the source. This system achieves the intelligent management for the storage and access for explosives and detonators, which can avoid the safety accidents from the greatest degree of caused by such sources.

\section{Acknowledgement}

In this paper, the research was sponsored by the Nature Science Foundation of Henan Province (Project No. 142300410286) and by the Nature Science Foundation of Luohe medical college(2013-S-LMC27).

\section{Reference}

[1] Chenwei Cai, Haijian, Fan, Biyang Liu.Design and Application of Gate Control System Based on Fingerprint Identification Technology [J] ,Video Engineering. 2012.36(3) 128-130

[2] Siemens TC35/TC37 Hardware Interface [EB/OL].http//www.siemens.com/entry/cc/ en/.

[3] Siemens.AT Command Set Siemens Cellular Engines.Convright(C)Siemens AG 2001.

[4] Pan Bin hong-xia guo. Short message transceiver module TC35i peripheral circuit design [J]. Journal of single-chip microcomputer and embedded system application, 2004, (7) : 38-41.

[5] Li. Using single-chip microcomputer control cell phone text message [J]. Journal of electronic technology applications, 2002 (1) : 24-26. 